\title{
Protein phosphatase 1 regulatory subunit 12A and catalytic subunit $\delta$, new members in the phosphatidylinositide 3 kinase insulin-signaling pathway
}

\author{
Thangiah Geetha ${ }^{1,+}$, Paul Langlais ${ }^{1}$, Michael Caruso ${ }^{2}$ and Zhengping Yi $^{1,2}$ \\ ${ }^{1}$ Center for Metabolic and Vascular Biology, Arizona State University, Tempe, Arizona 85287, USA \\ ${ }^{2}$ Department of Pharmaceutical Sciences, Room 3146, Eugene Applebaum College of Pharmacy/Health Sciences, Wayne State University, 259 Mack Avenue, \\ Detroit, Michigan 48201, USA \\ (Correspondence should be addressed to Z Yi at Department of Pharmaceutical Sciences, Wayne State University; Email: zhengping.yi@wayne.edu) \\ ${ }^{+}$( $T$ Geetha is now at Department of Nutrition, Dietetics and Hospitality Management, Auburn University, Auburn, Alabama, USA)
}

\begin{abstract}
Skeletal muscle insulin resistance is an early abnormality in individuals with metabolic syndrome and type 2 diabetes (T2D). Insulin receptor substrate-1 (IRS1) plays a key role in insulin signaling, the function of which is regulated by both phosphorylation and dephosphorylation of tyrosine and serine/threonine residues. Numerous studies have focused on kinases in IRS1 phosphorylation and insulin resistance; however, the mechanism for serine/threonine phosphatase action in insulin signaling is largely unknown. Recently, we identified protein phosphatase 1 (PP1) regulatory subunit 12A (PPP1R12A) as a novel endogenous insulin-stimulated interaction partner of IRS1 in L6 myotubes. The current study was undertaken to better understand PPP1R12A's role in insulin signaling. Insulin stimulation promoted an interaction between the IRS1/p85 complex and PPP1R12A; however, p85 and PPP1R12A did not interact independent of IRS1. Moreover, kinase
\end{abstract}

inhibition experiments indicated that insulin-induced interaction between IRS1 and PPP1R12A was reduced by treatment with inhibitors of phosphatidylinositide 3 kinase, PDK1, Akt, and mTOR/raptor but not MAPK. Furthermore, a novel insulin-stimulated IRS1 interaction partner, PP1 catalytic subunit $(\mathrm{PP} 1 \mathrm{c} \delta)$, was identified, and its interaction with IRS1 was also disrupted by inhibitors of Akt and $\mathrm{mTOR} /$ raptor. These results indicate that PPP1R12A and PP1c $\delta$ are new members of the insulinstimulated IRS1 signaling complex, and the interaction of PPP1R12A and PP1c $\delta$ with IRS1 is dependent on Akt and $\mathrm{mTOR} /$ raptor activation. These findings provide evidence for the involvement of a particular PP1 complex, $\mathrm{PPP} 1 \mathrm{R} 12 \mathrm{~A} / \mathrm{PP} 1 \mathrm{c} \delta$, in insulin signaling and may lead to a better understanding of dysregulated IRS1 phosphorylation in insulin resistance and T2D.

Journal of Endocrinology (2012) 214, 437-443

\section{Introduction}

Insulin regulates a wide variety of biological processes, and defects in insulin signaling in skeletal muscle are considered to be one of the main causes for a large number of disease conditions, such as insulin resistance, metabolic syndrome, type 2 diabetes (T2D), cardiovascular diseases, and cancer (Bouzakri et al. 2005, Abdul-Ghani \& DeFronzo 2010). The intracellular actions of insulin are mediated by posttranslational modifications of regulatory proteins (e.g. kinases and phosphatases), such as phosphorylation and dephosphorylation, through controlled protein-protein interactions. Two putative insulin-signaling pathways have emerged, the phosphatidylinositide 3 kinase (PI3K) and the MAPK-signaling pathways (Siddle 2011). Insulin receptor substrate-1 (IRS1) is a keystone mediator bridging insulin receptor (IR) activation to both the PI3K and MAPK insulinstimulated pathways (Valverde et al. 2001, Siddle 2011). IR-induced tyrosine phosphorylated IRS1 recruits p85 subunit of PI3K and leads to downstream activation of phosphoinositide-dependent kinase 1 (PDK1) and Akt. Activated Akt promotes GLUT4 translocation/glucose uptake through AS160, increases glycogen synthesis, and enhances protein synthesis through mammalian target of rapamycin (mTOR) bound to its regulatory protein, raptor (Siddle 2011). Moreover, IRS1 interacts with growth factor 
receptor binding protein 2 (GRB2), leading to MAPK activation and subsequent promotion of cell survival and mitogenesis (Valverde et al. 2001). A number of the downstream serine/threonine kinases, such as Akt, mTOR/ raptor, ribosomal protein S6 kinase beta-1 (S6K1), glycogen synthase kinase 3 (GSK3), and MAPK, have been shown to phosphorylate IRS1 on serine/threonine residues (Gual et al. 2005, Sun \& Liu 2009, Siddle 2011). The majority of these phosphorylation events have been shown to act as negative feedback signals to attenuate insulin signaling; consequently, elevated serine/threonine phosphorylation ( $\mathrm{pS} / \mathrm{T}$ ) of IRS1 is considered to be one of the main causes for insulin resistance and T2D (Gual et al. 2005, Sun \& Liu 2009, Siddle 2011). Recently, we provided a relative global assessment of IRS1 phosphorylation in vivo in humans (Langlais et al. 2011): basal IRS1 Ser303 and Ser323 phosphorylation was increased in muscle biopsies from T2D patients, while Thr495 phosphorylation was decreased in T2D. Moreover, insulin increased IRS1 phosphorylation at Ser527, Ser531, Ser636, and Ser1101 while suppressed Ser348 and Thr446 phosphorylation in both insulin-sensitive and -resistant human participants. These results show that insulin can both stimulate and suppress IRS1 pS/pT at specific sites in healthy humans and diabetic patients, suggesting that the activation of both kinases and phosphatases on IRS1 is needed for proper insulin signaling. However, the majority of research has been focused on IRS1 pS/pT kinases, and little is known about how serine/threonine phosphatases act on IRS1.

In our previous work, we identified protein phosphatase 1 (PP1) regulatory subunit $12 \mathrm{~A}$ (PPP1R12A) as a novel endogenous insulin-stimulated interaction partner of IRS1 in L6 myotubes (Geetha et al. 2011), an established insulinresponsive cell model. PPP1R12A, also known as myosin phosphatase target subunit 1 (MYPT1 or MBS), can bind to the catalytic subunit of PP1, PP1c $\delta$, to modulate the specificity and activity of $\mathrm{PP} 1 \mathrm{c} \delta$ against its substrates (Matsumura \& Hartshorne 2008, Grassie et al. 2011). PPP1R12A is widely expressed in many cell types and is highly expressed in smooth muscle cells (Matsumura \& Hartshorne 2008) as well as in mouse extensor digitorum longus muscle (EDL) and soleus muscles (Ryder et al. 2007). One function of PPP1R12A is to form a myosin phosphatase holoenzyme with PP1c $\delta$ in smooth muscle cells, thus modulating the specificity and activity of PP1c $\delta$ against phosphorylated myosin, which regulates subsequent muscle contraction and cell migration (Ito et al. 2004, Okamoto et al. 2006, Matsumura \& Hartshorne 2008, Grassie et al. 2011). In addition, several other proteins involved in different biological processes have been shown to interact with PPP1R12A, such as polo-like kinase 1, interleukin-16, and telokin, suggesting that PPP1R12A may be involved in various additional cell functions (Ito et al. 2004, Matsumura \& Hartshorne 2008, Grassie et al. 2011).

In this study, we first immunoprecipitated PPP1R12A and western blotted for IRS1 to confirm the interaction between endogenous PPP1R12A and IRS1 in L6 myotubes. Then we employed kinase inhibitor experiments to assess whether PPP1R12A is involved in the PI3K or MAPK insulin signaling pathway.

\section{Materials and Methods}

\section{Antibodies and reagents}

Anti-PPP1R12A, anti-PP1c, and anti-p-ERK/MAPK were purchased from Santa Cruz Biotechnology; anti-IRS1 from Millipore, Temecula, CA, USA; and anti-p85 was obtained from Upstate, Temecula, CA, USA. Anti-pSer473 for Akt, anti-Akt, anti-p-Thr378 for p70S6K, anti-p70S6K, and antip44/42 MAPK antibodies were purchased from Cell Signaling Technologies, Beverly, MA, USA. Wortmannin, PD98059, and rapamycin were purchased from Sigma; OSU03012 from Cayman Chemical, Ann Arbor, MI, USA; and Akt inhibitor (Akti-1/2) from Calbiochem (Billerica, MA, USA). Anti-rabbit IgG and anti-mouse IgG-HRPlinked secondary antibody were ordered from GE Healthcare UK Ltd. (Chalfont St Giles, Buckinghamshire, UK) and ECL from PerkinElmer, Shelton, CT, USA. DMEM medium was purchased from Invitrogen. Bradford reagent and nitrocellulose membranes were ordered from Bio-Rad. Protein A sepharose beads and all other reagents were obtained from Sigma.

\section{Cell culture}

The parental L6 cells were maintained in DMEM medium supplemented with $10 \%$ fetal bovine serum and penicillin/ streptomycin in a humidified atmosphere containing 5\% $\mathrm{CO}_{2}$ and $95 \%$ air at $37^{\circ} \mathrm{C}$. Cells were subcultured by trypsinization of subconfluent cultures using $0 \cdot 25 \%$ trypsin. L6 myoblasts were seeded at a density of $8 \times 10^{5}$ cells per $10 \mathrm{~cm}$ dish. For differentiation into myotubes, myoblasts were replaced with medium containing $2 \%$ fetal bovine serum and penicillin/streptomycin every $24 \mathrm{~h}$ for 7 days. The cells were deprived of serum in culture medium for $4 \mathrm{~h}$ at $37^{\circ} \mathrm{C}$ before cell lysis.

\section{Immunoprecipitation and western blotting analysis}

L6 myotubes were stimulated with or without insulin $(100 \mathrm{nM})$ for $15 \mathrm{~min}$ at $37^{\circ} \mathrm{C}$. The cells were lysed with $1 \mathrm{ml}$ lysis buffer (50 mM HEPES (pH 7·6), $150 \mathrm{mM}$ $\mathrm{NaCl}, 20 \mathrm{mM}$ sodium pyrophosphate, $10 \mathrm{mM} \mathrm{NaF}$, $20 \mathrm{mM}$ beta-glycerophosphate, $1 \%$ Triton, $1 \mathrm{mM} \mathrm{Na}_{3} \mathrm{VO}_{4}$, $1 \mathrm{mM}$ phenylmethylsulfonyl fluoride, and $10 \mu \mathrm{g} / \mathrm{ml} \mathrm{leu-}$ peptin and aprotinin). Protein was estimated by Bradford procedure. Cell lysates $(1 \mathrm{mg})$ were diluted in lysis buffer and incubated with $4 \mu \mathrm{g}$ primary antibody. The immunoprecipitates were collected with protein A agarose beads overnight at $4{ }^{\circ} \mathrm{C}$ and then were washed three times with PBS. 
Samples were boiled in SDS-PAGE buffer and resolved on 7-5-10\% SDS-PAGE, transferred onto nitrocellulose membranes, analyzed by western blotting with the appropriate antibodies, and the immune complex was detected by chemiluminescence.

\section{Results}

Insulin induces the interaction of IRS1 and p85 with PPP1R12A

We previously reported that IRS1 interacts with PPP1R12A upon insulin stimulation by HPLC-ESI-MS/MS and confirmed the interaction by immunoprecipitating IRS1 and western blotting for PPP1R12A (Geetha et al. 2011). In the present work, we further confirmed the interaction by immunoprecipitating PPP1R12A and western blotting for IRS1. L6 myotubes were stimulated with or without $100 \mathrm{nM}$ insulin for $15 \mathrm{~min}$ and immunoprecipitated with anti-PPP1R12A antibody followed by immunoblotting with antibodies specific to IRS1, PPP1R12A, and p85. The results indicate that the signal for interaction of IRS1 and p85 was enhanced upon insulin stimulation in the PPP1R12A immunoprecipitates (Fig. 1A) and clearly confirmed the insulin-stimulated association of PPP1R12A, IRS1, and p85. As it is well known that p85 is an insulin-stimulated IRS1 interaction partner, p85 might not bind to PPP1R12A directly and instead is co-immunoprecipitating with IRS1.

A

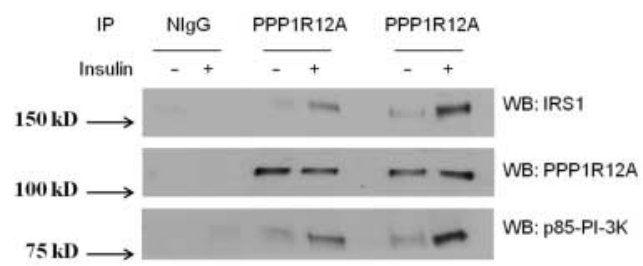

B

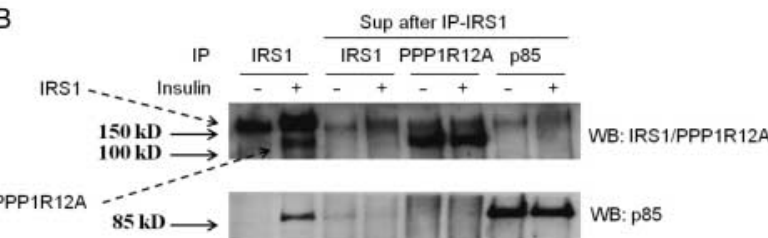

Figure 1 IRS1 and p85 regulatory subunit of PI3K interacts with PPP1R12A upon insulin stimulation. (A) L6 myotubes were serum starved for $4 \mathrm{~h}$ and stimulated with or without insulin (100 nM) for $15 \mathrm{~min}$ at $37^{\circ} \mathrm{C}$. The cells were lysed and $1 \mathrm{mg}$ lysates was immunoprecipitated (IP) with normal IgG or PPP1R12A antibody and western blotted (WB) with IRS1, PPP1R12A, and p85. (B) The supernatant, after immunoprecipitating twice with IRS1 antibody, was further IP with antibodies specific to IRS1, PPP1R12A, or p85 subunit as indicated. The immunoprecipitates were WB with IRS1/PPP1R12A double antibody and p85. Lanes 1 and 2 were whole-cell lysates IP with anti-IRS1 as positive control. All experiments are representative of three separate experiments. p85 and PPP1R12A do not interact independent of IRS1

The results from Fig. 1A suggest that insulin stimulates the formation of a complex between PPP1R12A, IRS1, and p85. To test whether p85 interacts with PPP1R 12A directly, we used supernatants that were immunoprecipitated twice with antiIRS1 antibody to deplete IRS1 from the sample. We then performed immunoprecipitation with the supernatants with either IRS1, PPP1R12A, or p85 antibody as indicated in Fig. 1B. As expected, IRS1 was substantially reduced in supernatants that were immunoprecipitated twice with antiIRS1 (Fig. 1B, lanes 3-8). The interaction between PPP1R2A and p 85 was abolished in the absence of IRS1 (Fig. 1B, lanes 5-8). Altogether, these findings reveal that IRS1, PPP1R12A, and $\mathrm{p} 85$ form a complex upon insulin stimulation, and p85 does not associate with PPP1R12A independent of IRS1.

\section{The interaction between PPP1R12A and IRS1 is dependent on PI3K activity}

As PI3K and MAPK are two major pathways in insulin signaling, we next wanted to explore whether PI3K or MAPK activity is required for the insulin-stimulated interaction of PPP1R12A with IRS1. L6 myotubes were pretreated with either $50 \mathrm{nM}$ wortmannin, a PI3K inhibitor (Castillo et al. 2006, Codina et al. 2008), or $50 \mu \mathrm{M}$ PD98059, a MAPK inhibitor (Coogan et al. 1999), for $1 \mathrm{~h}$ followed by insulin stimulation $(100 \mathrm{nM})$ for $15 \mathrm{~min}$. The interaction between PPP1R12A and IRS1 was examined by immunoprecipitating the lysates with an IRS1 antibody and western blotting with anti-IRS1, PPP1R12A, and p85 (Fig. 2A). Wortmannin disrupted the interaction between PPP1R12A and IRS1 whereas PD98059 treatment did not affect the PPP1R12A/ IRS1 interaction. These results imply that activation of $\mathrm{PI} 3 \mathrm{~K}$, but not MAPK, is required for the insulin-induced interaction of PPP1R12A with IRS1. To ensure efficient insulin stimulation and either wortmannin or PD98059 inhibition of the PI3K and MAPK pathways, respectively, the whole cell lysates were probed for the phosphorylation of either Akt or MAPK (Fig. 2B). Treatment of either wortmannin or PD98059 attenuated but did not abolish insulin-stimulated phosphorylation of Akt and MAPK phosphorylation respectively.

\section{The activity of PDK1 is required for the interaction of PPP1R12A with IRS1}

As the interaction between PPP1R12A and IRS1 is dependent on PI3K activity, and the kinase PDK1 is downstream of PI3K, the next step was to analyze whether PDK1 activation is required for the PPP1R12A/IRS1 interaction. L6 myotubes were pretreated with a PDK1 inhibitor (10 $\mu \mathrm{M}$ of OSU03012) (Porchia et al. 2007) for $4 \mathrm{~h}$ after serum starvation followed by insulin stimulation. Cells were lysed and the PPP1R12A/IRS1 interaction was analyzed by immunoprecipitation with IRS1 antibody and western 


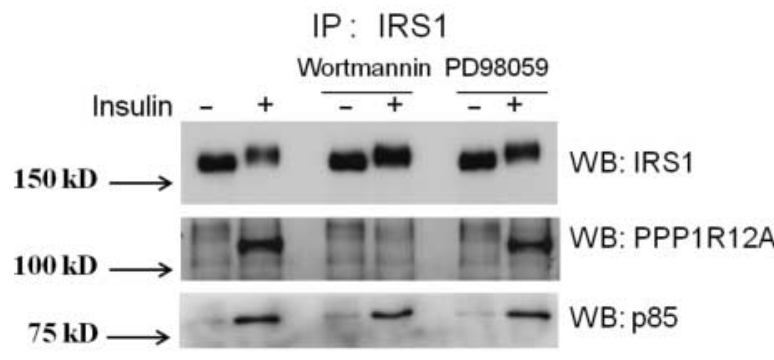

IP: IRS1

OSU03012

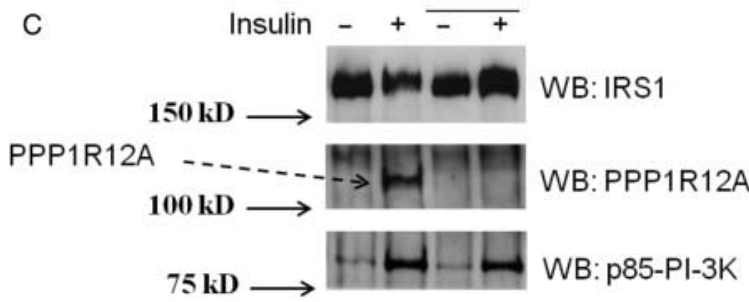

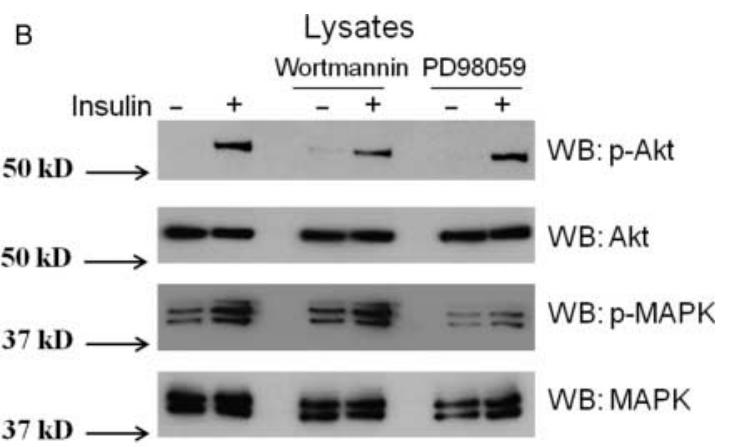

D

Lysates

OSU03012

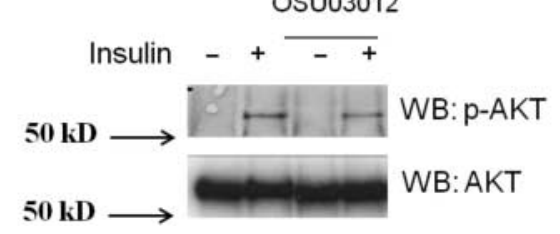

Figure 2 The insulin-stimulated interaction of PPP1R12A and IRS1 was disrupted upon inhibition of PI3K and PDK1 activity. (A) L6 myotubes were pretreated with the PI3K inhibitor (50 nM wortmannin for $1 \mathrm{~h}$ ) or MAPK inhibitor (50 $\mu \mathrm{M}$ PD98059 for $1 \mathrm{~h}$ ) followed by insulin stimulation $(100 \mathrm{nM})$ for $15 \mathrm{~min}$. Co-interaction was examined by immunoprecipitating (IP) the lysates with IRS1 antibody and western blotting (WB) with anti-IRS1, PPP1R12A, and p85; (B) equivalent protein from cell lysates (30 $\mu$ g) from the above was immunoblotted (WB) with phosphor-Akt antibody that recognizes the phosphorylation at serine 473, Akt, phosphor-MAPK, and MAPK antibody; (C) myotubes were treated with $10 \mu \mathrm{M}$ OSU03012, a PDK1 inhibitor, for $4 \mathrm{~h}$ after serum starvation followed by $100 \mathrm{nM}$ insulin stimulation for $15 \mathrm{~min}$. Cells were lysed and immunoprecipitated with IRS1 antibody and immunoblotted with IRS1, PPP1R12A, and p85 antibody; (D) the cell lysates were western blotted (WB) with phosphor-Akt and Akt antibody. All experiments are representative of three separate experiments.

blotting with IRS1, PPP1R12A, or p85 antibody (Fig. 2C). The PDK1 inhibitor reduced the interaction between PPP1R12A and IRS1, indicating that PDK1 activation is required for the interaction between PPP1R12A and IRS1. In addition, the lysates were blotted for phosphor-Akt and Akt antibody to confirm the inhibitory effect of the PDK1 inhibitor, which attenuated the Akt phosphorylation at phospho-Ser473 (pS473) on insulin stimulation compared with the control lysates (Fig. 2D). It is noted that we did not perform the experiments regarding phospho-Thr308 (pT308), a well-known PDK1 phosphorylation site; however, based on the literature, it is most likely that Thr308 would show similar inhibition of phosphorylation as pS473 upon the treatment of OSU03012 (Lee et al. 2009). It has been shown that OSU03012 treatment decreased both pS473 and pT308 of Akt in human malignant schwannoma HMS-97 cells and primary human vestibular schwannoma cells (Lee et al. 2009).

Activation of Akt and $m T O R /$ raptor is required for the insulin-stimulated interaction of PPP1R $12 A$ and $P P 1 c \delta$ with IRS1

As described earlier, Akt is downstream of PDK1, and $\mathrm{mTOR} /$ raptor is downstream of Akt in the insulin signaling pathway. To identify whether the activation of Akt or
$\mathrm{mTOR} /$ raptor is necessary for the insulin-stimulated interaction of PPP1R12A with IRS1, L6 myotubes were treated with $500 \mathrm{nM}$ Akt inhibitor, Akti (Fazakerley et al. 2010), for $1 \mathrm{~h}$ or $50 \mathrm{nM}$ mTOR inhibitor, rapamycin (Haruta et al. 2000), for $30 \mathrm{~min}$, followed by insulin stimulation. IRS1 was immunoprecipitated followed by western blotting with anti-IRS1, PPP1R12A, and p85 (Fig. 3A). The interaction of PPP1R12A and IRS1 was blocked by both the Akt inhibitor and rapamycin, suggesting that Akt and $\mathrm{mTOR} /$ raptor activity is necessary for the interaction. To verify Akti and rapamycin inhibition of Akt and mTOR activity, the lysates were probed for the phosphorylation of Akt and the mTOR substrate p70S6K respectively (Fig. 3B). Phosphorylation of both Akt and $\mathrm{p} 70 \mathrm{~S} 6 \mathrm{~K}$ was diminished following inhibition compared with control. Akti has been shown to block both basal and insulin-stimulated phosphorylation and activation of Akt in L6 myotubes (Fazakerley et al. 2010). Furthermore, we also western blotted for PP1c $\delta$ in the IRS1 immunoprecipitates and found that PP1c $\delta$ has an increased binding to IRS1 upon insulin stimulation (Fig. 3A). It is well known that PPP1R12A can bind to PP1c $\delta$; therefore, it is likely that PP1c $\delta$ interact with IRS1 indirectly through PPP1R12A. Nonetheless, this result provided Co-IP evidence that a catalytic subunit of a serine/threonine phosphatase may interact with IRS1. 
A

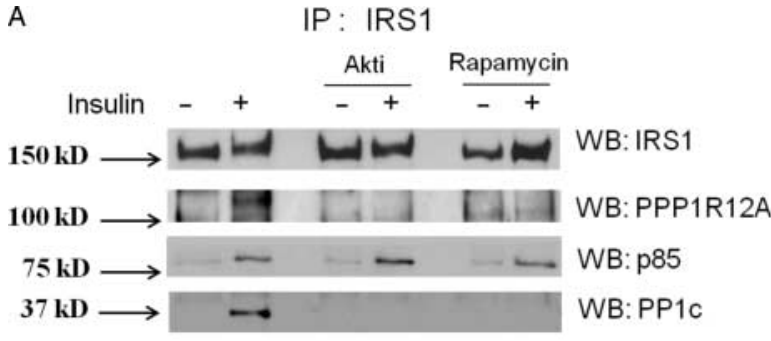

B

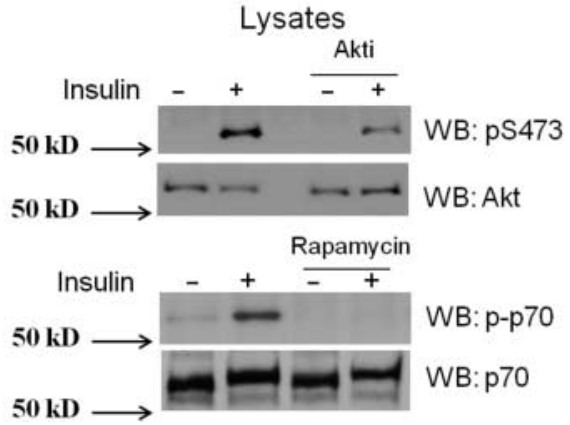

Figure 3 Activity of Akt and mTOR/raptor is required for the insulin-stimulated interaction of PPP1R12A and PP1 $\delta$ with IRS1. (A) $500 \mathrm{nM}$ of the Akt inhibitor, Akti, was added to the L6 myotubes for $1 \mathrm{~h}$ or $50 \mathrm{nM}$ of the mTOR inhibitor, rapamycin, was added to the L6 myotubes for $30 \mathrm{~min}$ and followed by $100 \mathrm{nM}$ insulin stimulation for 15 min. IRS1 was immunoprecipitated (IP) followed by western blotting (WB) with anti IRS1, PPP1R12A, and p85; (B) equal protein from cell lysates was immunoblotted with anti-phosphor-Akt, Akt, phosphor, and non-phospho-p70.

All experiments are representative of three separate experiments.

\section{Discussion}

Posttranslational modifications of proteins such as phosphorylation and dephosphorylation are known mechanisms that operate as a 'molecular switch' to either promote or diminish insulin signaling. Once the IR is activated and appropriate tyrosine residues of IRS1 are phosphorylated (Venable et al. 2000), IRS1 interacts with p85 and Grb2, which are affiliated with the PI3K and MAPK pathways, respectively, leading to the activation of a number of downstream kinases, such as Akt/PKB, mTOR, S6K1, GSK3, and MAPK. These downstream kinases can phosphorylate site-specific serine/threonine residues of IRS1, in which most of these phosphorylation events result in reduced insulin action (Gual et al. 2005, Sun \& Liu 2009, Siddle 2011). Surprisingly, little is known about the role of serine/ threonine phosphatases in IRS1 phosphorylation and insulin-signaling transduction.

In the current study, we demonstrate a regulatory subunit and a catalytic subunit of a serine/threonine phosphatase, PPP1R12A and PP1c $\delta$, as new members of the insulinsignaling pathway in L6 myotubes. Insulin stimulation promotes an interaction between the IRS1/p85 complex and PPP1R12A; however, p85 and PPP1R12A do not interact independent of IRS1 (Fig. 1). Kinase inhibitor experiments suggest that insulin-stimulated interaction between PPP1R12A and IRS1 is contingent on the activation of PI3K, PDK1, Akt/PKB, and mTOR/raptor (mTORC1) but not MAPK (Figs 2 and 3). In addition, insulin-stimulated interaction between PP1c $\delta$ and IRS1 is contingent on the activation of Akt/PKB and mTORC1 (Fig. 3). Whether the insulin-stimulated interaction between PPP1R $12 \mathrm{~A} / \mathrm{PP} 1 \mathrm{c} \delta$ and IRS1 is mediated directly by mTORC1 and/or other downstream signaling molecules (e.g. S6K1) remains undetermined. The activation of mTORC1 for PPP1R12A and $\mathrm{PP} 1 \mathrm{c} \delta$ association with IRS1 suggests a positive regulatory feedback role involving mTOR's actions: protein synthesis, cell survival, cell differentiation, and increased transcription (Proud 2011). However, mTOR-dependent insulin-stimulated PPP1R12A/IRS1 interaction might extend to positive feedback for downstream molecules of IRS1, which may affect mitogenic and metabolic processes including glucose uptake and glycogen synthesis. It is possible that activated mTOR/ raptor upon insulin stimulation activates PPP1R12A, enabling PPP1R12A to anchor PP1c $\delta$ to dephosphorylate IRS1. As discussed before, insulin may activate a number of serine/ threonine kinases to phosphorylate IRS1, leading to reduced insulin action through IRS1. The main purpose of activation of the PPP1R12A/PP1c $\delta$ complex to dephosphorylate IRS1 may be to counterbalance the effect of insulin-activated serine/threonine kinases, resulting in balanced IRS1 pS/pT and proper insulin action through IRS1.

It is noted that even though the inhibitors of PI3K and PDK1 (wortmannin and OSU03012) did not completely abolish the phosphorylation of Akt (S473), it completely inhibited the association of PPP1R12A with IRS1. This indicates that the interaction between IRS1 and PPP1R12A is very sensitive to PI3K and PDK1 inhibition, and full activity of PI3K and PDK1 may be required for this

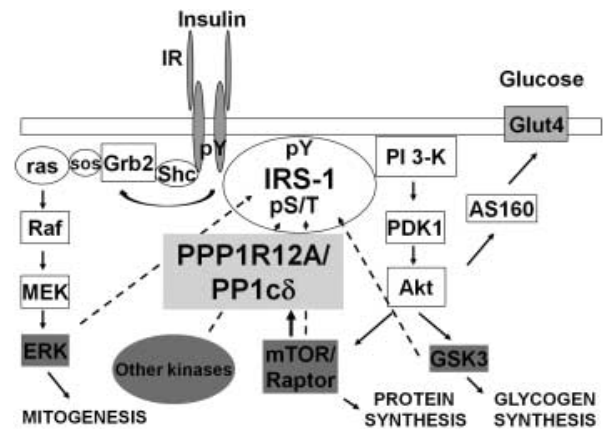

Figure $4 \mathrm{~A}$ model depicting PPP1R12A/PP1c $\delta$ in the insulin signaling pathway. Insulin stimulation leads to the activation of PI3K, PDK1, Akt, and mTOR/Raptor, as well as PPP1R12A. This leads to increased interaction between IRS-1 and PPP1R12A/PP1c $\delta$ and the ability of the PPP1R12A/PP1 $\delta \delta$ complex to dephosphorylate IRS-1. The main purpose of activation of the PPP1R12A/PP1c $\delta$ complex to dephosphorylate IRS1 may be to counterbalance the effect of insulin-activated serine/threonine kinases, resulting in balanced IRS1 pS/pT and proper insulin action through IRS1. 
interaction to occur. In addition, even though kinase inhibitors, such as wortmannin, have been used widely in studies of insulin signaling, they are problematic with regard to their specificity. Using only one kinase inhibitor might not be sufficient to define the pathway dependency of PPP1R12A. Therefore, we performed inhibitor experiments following the classic PI3K pathway, namely PI3K, PDK1, Akt, and $\mathrm{mTOR} /$ raptor, and each inhibition disrupted the interaction between IRS1 and PPP1R12A, thus providing supporting evidence for each other.

On the other hand, these disruptions had little effect on the association of IRS1 with p85. One of the possible explanations is that wortmannin or OSU03012 inhibition will result in the inactivation of downstream serine/threonine kinases, such as Akt/PKB, mTOR, S6K1, GSK3, etc. As we discussed earlier, these kinases have been shown to be able to phosphorylate IRS1 on serine/threonine, which may lead to diminished IRS1/p85 association and reduced insulin signaling. Based on the results from the current study, we hypothesized that PPP1R12A might play a positive role in insulin signaling by anchoring PP1c $\delta$ to dephosphorylate IRS1 and counterbalance the effect of these serine/threonine kinases on IRS1. After wortmannin or OSU03012 inhibition, these downstream serine/threonine kinases of PI3K and PDK1 may lose their negative effect on IRS1/p85 association, while PPP1R12A may lose its positive effect on IRS1/p85 association, resulting in little effect of wortmannin/ OSU03012 inhibition on IRS1/p85 association. In addition, PPP1R12A might affect the activity or subcellular localization of the IRS1/PI3K complex or regulate other interacting proteins involved in the formation of this important insulinsensitive protein complex. We are currently in the process of performing experiments to test these hypotheses, such as lentiviral shRNA-mediated PPP1R12A knockdown in L6 myotubes that may provide additional evidence on whether PPP1R12A regulates the interaction between IRS1 and p85. Recently, we have reported that multiple phosphorylation sites in PPP1R12A were either stimulated or suppressed by insulin (Chao et al. 2012). The findings that insulin regulates both PPP1R12A phosphorylation and the interaction of PPP1R12A with IRS1 strongly implicate a role for PPP1R12A in insulin action.

Previous reports have shown that treatment of fat cells or 3T3-L1 adipocytes with okadaic acid increases serine phosphorylation of IRS1 and results in reduced insulinstimulated IRS1 tyrosine phosphorylation (Tanti et al. 1994, Mothe \& Van Obberghen 1996). However, it was not clear whether PP1 is the phosphatase acting on IRS1, as okadaic acid inhibits both PP1 and the protein phosphatase 2A (PP2A). Furthermore, four mammalian PP1 catalytic subunits $\mathrm{PP} 1 \mathrm{c} \alpha, \mathrm{PP} 1 \mathrm{c} \beta$ (also called PP1c $\delta$ ), PP1c $\gamma 1$, and PP1c $\gamma 2$, with more than $100 \mathrm{PP} 1$ regulatory subunits (PP1R), have been identified (Virshup \& Shenolikar 2009, Bollen et al. 2010). The interactions between PP1c and PP1R lead to the formation of numerous PP1 complexes with unique substrate specificity, distinct subcellular localization, and various regulatory mechanisms. The present work provides evidence for the involvement of a particular PP1 complex, PPP1R12A/PP1c $\delta$, in the insulin-stimulated IRS1 signaling complex. These findings may lead to a better understanding of serine/threonine phosphatase regulation of IRS1 pS/pT and downstream insulin signaling.

To summarize the results from this study, we propose a model (Fig. 4) illustrating the presence of PPP1R12A/PP1c $\delta$ in the insulin-signaling pathway. Insulin stimulation leads to the activation of PI3K, PDK1, Akt, and mTOR/raptor. Meanwhile, insulin stimulation promotes an interaction between the IRS1/p85 complex and PPP1R12A (Fig. 1). In addition, inhibition of PI3K and PDK1 activity by wortmannin and OSU03012, respectively, abolished the insulin-stimulated interaction between PPP1R12A and IRS1 (Fig. 2). Moreover, as shown in Fig. 3, pretreatment of the cells with Akti and rapamycin to deactivate Akt and $\mathrm{mTOR} /$ raptor resulted in diminished insulin-stimulated interaction between PPP1R12A/PP1c $\delta$ and IRS1. One possible purpose of the PPP1R12A/PP1c $\delta$ complex interaction with IRS1 is to dephosphorylate IRS1 and to counterbalance the effect of insulin-activated serine/threonine kinases, resulting in balanced IRS1 $\mathrm{pS} / \mathrm{pT}$ and proper insulin action through IRS1. Dysfunctional PPP1R12A/ PP1c $\delta$ complex may contribute to the development of insulin resistance and T2D. Future experiments will concentrate on the interaction between PPP1R12A/PP1c $\delta$ and IRS1 in both animal and human models, while protein overexpression and/or siRNA-mediated protein knockdown will be employed to assess PPP1R12A/PP1c $\delta$ in IRS1 dephosphorylation and insulin signaling within in vitro insulinsignaling models, such as L6 myotubes. Elucidating the function of PPP1R12A/PP1c $\delta$ in insulin action may provide novel insights into serine/threonine phosphatase regulation of IRS1 and downstream insulin signaling, facilitating the understanding of the role of phosphatases in insulin action in health and disease.

\section{Declaration of interest}

The authors declare that there is no conflict of interest that could be perceived as prejudicing the impartiality of the research reported.

\section{Funding}

This work was supported by funds from the National Institute of Health R01DK081750 (Z Y).

\section{References}

Abdul-Ghani MA \& DeFronzo RA 2010 Pathogenesis of insulin resistance in skeletal muscle. Journal of Biomedicine \& Biotechnology 2010476279.

Bollen M, Peti W, Ragusa MJ \& Beullens M 2010 The extended PP1 toolkit: designed to create specificity. Trends in Biochemical Science 35 450-458. (doi:10.1016/j.tibs.2010.03.002) 
Bouzakri K, Koistinen HA \& Zierath JR 2005 Molecular mechanisms of skeletal muscle insulin resistance in type 2 diabetes. Current Diabetes Reviews 1 167-174. (doi:10.2174/1573399054022785)

Castillo J, Ammendrup-Johnsen I, Codina M, Navarro I \& Gutierrez J 2006 IGF-I and insulin receptor signal transduction in trout muscle cells. American Journal of Physiology. Regulatory, Integrative and Comparative Physiology 290 R1683-R1690. (doi:10.1152/ajpregu.00294.2005)

Chao A, Zhang X, Ma D, Langlais P, Luo M, Mandarino LJ, Zingsheim M, Pham K, Dillon J \& Yi Z 2012 Site-specific phosphorylation of protein phosphatase 1 regulatory subunit $12 \mathrm{~A}$ stimulated or suppressed by insulin. Journal of Proteomics 75 3342-3350. (doi:10.1016/j.jprot.2012.03.043)

Codina M, de la Serrana DG, Sanchez-Gurmaches J, Montserrat N, Chistyakova O, Navarro I \& Gutierrez J 2008 Metabolic and mitogenic effects of IGF-II in rainbow trout (Oncorhynchus mykiss) myocytes in culture and the role of IGF-II in the PI3K/Akt and MAPK signalling pathways. General and Comparative Endocrinology 157 116-124. (doi:10.1016/j.ygcen. 2008.04.009)

Coogan AN, O'Leary DM \& O’Connor JJ 1999 P42/44 MAP kinase inhibitor PD98059 attenuates multiple forms of synaptic plasticity in rat dentate gyrus in vitro. Journal of Neurophysiology 81 103-110.

Fazakerley DJ, Holman GD, Marley A, James DE, Stockli J \& Coster ACF 2010 Kinetic evidence for unique regulation of GLUT4 trafficking by insulin and AMP-activated protein kinase activators in L6 myotubes. Journal of Biological Chemistry 285 1653-1660. (doi:10.1074/jbc.M109.051185)

Geetha T, Langlais P, Luo M, Mapes R, Lefort N, Chen SC, Mandarino LJ \& Yi Z 2011 Label-free proteomic identification of endogenous, insulinstimulated interaction partners of insulin receptor substrate-1. Journal of the American Society for Mass Spectrometry 22 457-466. (doi:10.1007/s13361010-0051-2)

Grassie ME, Moffat LD, Walsh MP \& Macdonald JA 2011 The myosin phosphatase targeting protein (MYPT) family: a regulated mechanism for achieving substrate specificity of the catalytic subunit of protein phosphatase type 1delta. Archives of Biochemistry and Biophysics 510 147-159. (doi:10. 1016/j.abb.2011.01.018)

Gual P, Le Marchand-Brustel Y \& Tanti JF 2005 Positive and negative regulation of insulin signaling through IRS-1 phosphorylation. Biochimie $\mathbf{8 7}$ 99-109. (doi:10.1016/j.biochi.2004.10.019)

Haruta T, Uno T, Kawahara J, Takano A, Egawa K, Sharma PM, Olefsky JM \& Kobayashi M 2000 A rapamycin-sensitive pathway down-regulates insulin signaling via phosphorylation and proteasomal degradation of insulin receptor substrate-1. Molecular Endocrinology 14 783-794. (doi:10.1210/me.14.6.783)

Ito M, Nakano T, Erdodi F \& Hartshorne DJ 2004 Myosin phosphatase: structure, regulation and function. Molecular and Cellular Biochemistry 259 197-209. (doi:10.1023/B:MCBI.0000021373.14288.00)

Langlais P, Yi Z, Finlayson J, Luo M, Mapes R, De Filippis E, Meyer C, Plummer E, Tongchinsub P, Mattern M et al. 2011 Global IRS-1 phosphorylation analysis in insulin resistance. Diabetologia 54 2878-2889. (doi:10.1007/s00125-011-2271-9)

Lee TX, Packer MD, Huang J, Akhmametyeva EM, Kulp SK, Chen CS, Giovannini M, Jacob A, Welling DB \& Chang LS 2009 Growth inhibitory and anti-tumour activities of OSU-03012, a novel PDK-1 inhibitor, on vestibular schwannoma and malignant schwannoma cells. European Journal of Cancer 45 1709-1720. (doi:10.1016/j.ejca.2009.03.013)
Matsumura F \& Hartshorne DJ 2008 Myosin phosphatase target subunit: many roles in cell function. Biochemical and Biophysical Research Communications 369 149-156. (doi:10.1016/j.bbrc.2007.12.090)

Mothe I \& Van Obberghen E 1996 Phosphorylation of insulin receptor substrate- 1 on multiple serine residues, 612, 632, 662, and 731, modulates insulin action. Journal of Biological Chemistry 271 11222-11227. (doi:10.1074/jbc.271.19.11222)

Okamoto R, Kato T, Mizoguchi A, Takahashi N, Nakakuki T, Mizutani H, Isaka N, Imanaka-Yoshida K, Kaibuchi K, Lu Z et al. 2006 Characterization and function of MYPT2, a target subunit of myosin phosphatase in heart. Cell Signalling 18 1408-1416. (doi:10.1016/j.cellsig.2005.11.001)

Porchia LM, Guerra M, Wang YC, Zhang Y, Espinosa AV, Shinohara M, Kulp SK, Kirschner LS, Saji M, Chen CS et al. 2007 2-Amino-N-\{4-[5(2-phenanthrenyl)-3-(trifluoromethyl)-1H-pyrazol-1-yl]-phe nyl $\}$ acetamide (OSU-03012), a celecoxib derivative, directly targets p21-activated kinase. Molecular Pharmacology 72 1124-1131. (doi:10.1124/mol.107. 037556)

Proud CG 2011 mTOR signalling in health and disease. Biochemical Society Transactions 39 431-436. (doi:10.1042/BST0390431)

Ryder JW, Lau KS, Kamm KE \& Stull JT 2007 Enhanced skeletal muscle contraction with myosin light chain phosphorylation by a calmodulinsensing kinase. Journal of Biological Chemistry 282 20447-20454. (doi:10.1074/jbc.M702927200)

Siddle K 2011 Signalling by insulin and IGF receptors: supporting acts and new players. Journal of Molecular Endocrinology 47 R1-R10. (doi:10.1530/ JME-11-0022)

Sun XJ \& Liu F 2009 Phosphorylation of IRS proteins Yin-Yang regulation of insulin signaling. Vitamins and Hormones 80 351-387.

Tanti JF, Gremeaux T, van Obberghen E \& Le Marchand-Brustel Y 1994 Serine/threonine phosphorylation of insulin receptor substrate 1 modulates insulin receptor signaling. Journal of Biological Chemistry 269 6051-6057.

Valverde AM, Mur C, Pons S, Alvarez AM, White MF, Kahn CR \& Benito M 2001 Association of insulin receptor substrate 1 (IRS-1) y895 with Grb-2 mediates the insulin signaling involved in IRS-1-deficient brown adipocyte mitogenesis. Molecular and Cellular Biology 21 2269-2280. (doi:10.1128/ MCB.21.7.2269-2280.2001)

Venable CL, Frevert EU, Kim YB, Fischer BM, Kamatkar S, Neel BG \& Kahn BB 2000 Overexpression of protein-tyrosine phosphatase-1B in adipocytes inhibits insulin-stimulated phosphoinositide 3-kinase activity without altering glucose transport or Akt/Protein kinase B activation. Journal of Biological Chemistry 275 18318-18326. (doi:10.1074/jbc.M908392199)

Virshup DM \& Shenolikar S 2009 From promiscuity to precision: protein phosphatases get a makeover. Molecular Cell 33 537-545. (doi:10.1016/ j.molcel.2009.02.015)

Received in final form 21 June 2012

Accepted 22 June 2012

Made available online as an Accepted Preprint 22 June 2012 